\title{
ATLAS: the Laser Tomographic Adaptive Optics module for the E-ELT
}

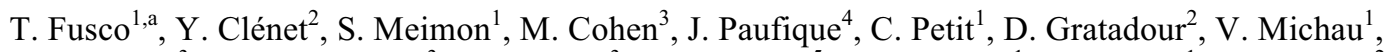
J.-P. Amans ${ }^{3}$, J.-L. Dournaux ${ }^{3}$, P. Jagourel ${ }^{3}$, H. Schnetler ${ }^{5}$, J.-M. Conan ${ }^{1}$, C. Robert ${ }^{1}$, E. Gendron ${ }^{2}$, G. Rousset ${ }^{2}$, N. Hubin ${ }^{4}$

${ }^{1}$ ONERA, Optics Department, 29 av. de la division Leclerc, 92322, Châtillon, France

${ }^{2}$ Observatoire de Paris Meudon, LESIA, 5 place Jules Janssen, 92190, Meudon, France

${ }^{3}$ Observatoire de Paris Meudon, GEPI, 5 place Jules Janssen, 92190, Meudon, France

${ }^{4}$ ESO, Karl Schwarzschildstr. 2, D-85748, Garching, Germany

${ }^{5}$ UK-Astronomy Technology Center, Royal Observatory, Blackford Hill, Edinburgh, EH9, 3HJ

\begin{abstract}
ATLAS is the future Laser Tomographic AO system of the E-ELT. Its goal is to provide a diffraction limited PSF in K band for a significant part of the sky (typically more than $60 \%$ ). A first concept of the module combined with optomechanical implementation and associated performance is presented. Results show the feasibility of the concept, its versatility and a relative simplicity which is a good first step toward a potential implementation in the early time of the telescope.
\end{abstract}

\section{Introduction}

ATLAS (Advanced Tomography with Laser for AO system) is the LTAO module for the E-ELT. Its purpose is to perform a high level on-axis correction of atmospheric turbulence using the telescope adaptive M4 $[1,2]$ and the field stabilization correction provided by the telescope M5 [3]. From the wavefront sensing point of view, the LTAO module includes multiple LGS wavefront sensors for high-order mode sensing and two NGS wavefront sensors, to measure the low-order modes. The NGS wavefront sensor sensitivity and performance will determine the sky coverage of the module. The LTAO module can be considered as a next step after a classical Single Conjugated AO system: by using several Laser Guide Stars, the module increases the sky coverage and compensates for the large cone effect due to the E-ELT size. The LTAO module is intended as a complementary AO facility with respect to the GLAO mode (wide field with moderate correction, at the level of a seeing improvement) and to the MCAO module (large FoV and moderate level of correction [4]). ATLAS system has several potential clients (scientific instruments) :

- HARMONI: a project of a single field, wide band and integral field spectrograph for the E-ELT, led by the Oxford University (PI: N. Thatte) and gathering the UK Astronomy Technological Centre (UK), the Instituto de Astrofísica de Canarias (Spain), the Centre de Recherche Astrophysique de Lyon (France) and the Consejo Superior de Investigaciones Cientificas (Spain).

- METIS a project of a mid-infrared imaging and spectroscopic instrument for the E-ELT led by the Leiden Observatory (PI: B. Brandl) and gathering the MPIA Heidelberg (Germany), the CEA Saclay (France), the UK Astronomy Technological Centre (UK) and the KU Leuven (Belgium).

- SIMPLE a project of a cryogenic high resolution near-infrared spectrometer for the E-ELT led by INAF Bologna (PI: L. Origlia) and gathering INAF Arcetri (Italy), INAF Roma (Italy), the UPPSALA Universitet (Sweden), the Pontifica Universidad Católica de Chile and the Thüringer Landessternwarte Tautenburg (Germany).

a e-mail : thierry.fusco@onera.fr

This is an Open Access article distributed under the terms of the Creative Commons Attribution-Noncommercial License, which permits unrestricted use, distribution, and reproduction in any noncommercial medium, provided the original work is properly cited. 


\section{System Overview}

The main specifications of ATLAS is to reach a $50 \%$ SR in $\mathrm{K}$ band for median seeing conditions (as defined by ESO, that is $\left.0.8^{\prime \prime}\right)$ with an associated sky coverage larger than $60 \%$ at a $60^{\circ}$ galactic latitude. It also has to provide a stable PSF (in position) with a 2 mas rms accuracy. Finally, ATLAS should ensure a FWHM PSF smaller than 17 mas for the whole sky.

Figure 1 gives an overview of the ATLAS system both in terms of functionalities and overall control scheme. We just recall here the main high level characteristics of ATLAS:

a 30" (in diameter) completely un-obscured and free from optics scientific FoV

a less than $4 \mathrm{~m}$ diameter $\mathrm{x} 1 \mathrm{~m}$ thick bonnette completely linked to the pre-focal station and thus rotating with the field (allowing the possibility to support a light instrument)

6 LGS system for HO measurements, LGSs being fixed w.r.t. the sky.

2 NGS IR ${ }^{\mathrm{b}}$-WFS to be picked off in a $30 " \rightarrow 2$ arcmin diameter FoV in order to have a good sky coverage (typically 50\%@ galactic pole)

- a dedicated calibration concept allowing to optically link the ATLAS focal plane and the scientific instrument focal plane in order to ensure a very stable PSF $(<2$ mas) at the scientific focus

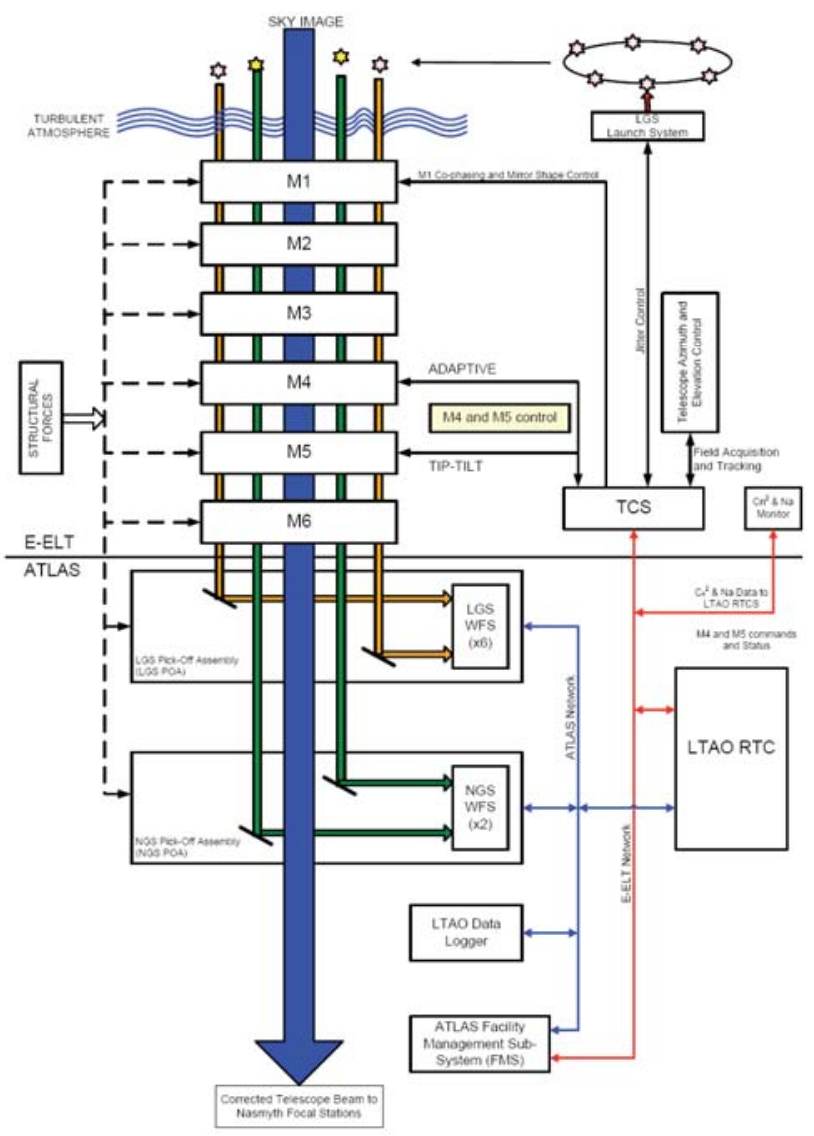

Figure 1 Schematic view of ATLAS and its environment

${ }^{\mathrm{b}} \mathrm{H}$ band 


\subsection{LGS positions and numbers}

The main purpose of the multi-GS tomographic reconstruction is to compensate for the LGS cone effect. For that purpose, Laser GS have to be located far from the optical axis. It is therefore clear that there will be a combination of two effects:

- Reduction of the unseen regions (through a better coverage of the cylindrical part of the turbulence associated to the scientific object wavefront)

- Increase of residual anisoplanatism error (due to the distance between LGS)

For ATLAS configuration, 6 LGS located on a 4.3 arcmin diameter ring have been considered. This solution represents the result of a complex trade-off between tomographic reconstruction performance, size of the NGS FoV (for sky coverage optimisation), scientific requirements (no optics in the scientific path), and overall opto-mechanical design.

\subsection{NGS numbers and WFS scheme}

The instrument residual tip-tilt requirement is 2 mas rms. The windshake contribution to Tip-tilt perturbation is worth $\sim 300$ mas rms (while turbulence contribution to tip-tilt is $\sim 10$ mas rms, due to the very favourable L0/D ratio). In other words, Atlas will rely on Natural Guide Stars to reduce by a factor 150 the Tip Tilt perturbation, which is essentially due to Windshake. To preserve reasonable sky coverage, each photon coming from the NGS to sense Tip Tilt has to be optimally exploited. That means a smart control law, a low detection noise, a concentration of the photons onto a small patch and a wave front sensor (WFS) concept with favourable noise propagation.

- Control law

The windshake signal is highly temporally correlated (half decorrelation time $=0.2 \mathrm{~s}$ ). It is therefore possible and very beneficial to predict next values of the Tip Tilt. The rejection obtained with a Kalman predictive filter is higher than classical integrator's by a factor 3 (in rms). With a Kalman filter, the optimal frame rate is around $500 \mathrm{~Hz}$.

- Noise

In addition to photon and detector noise, sky background noise limits the upper value of the WFS central wavelength to 2.2 microns ( $\mathrm{K}$ short). An additional analysis shows that, in absence of cryogenic envelope for ATLAS, the thermal emission for a $0^{\circ} \mathrm{C}$ ambient temperature yields $\sim 100$ photon per pixel per integration time in Ks band. This level of background noise is not acceptable. A cryogenic envelope would be heavy and complex, and is not considered in the current baseline, so the WFS central wavelength at this stage is either in the visible or in the $\mathrm{J} / \mathrm{H}$ band.

- Concentration of the photons

To optimize wave front sensing, one must concentrate the maximum number of photons within the zone of the detector used for computation. Additionally, this zone should be as small as possible to limit detector noise. In the case of small aberrations (other than Tip Tilt), this zone is restricted to the central patch of the Airy pattern. The portion of the total flux included in this zone can be approximated by the Strehl Ratio. In other words, to optimize WFS performance, one must maximize the number of photons at the entrance of the WFS, and maximize the SR at the level of the detector. The "efficient photons" are the one that do not spread the focal spot. The WFS measurement noise evolves in (Nphot x SR)-1 in photon noise regime, and in (Nphot x SR)-2 in detector noise regime. This criterion will help to select the WFS spectral band:An IR WFS is by far preferable to a visible one, because the SR in IR () is much higher than the SR in the visible. For the same reason, the potential gain of including $\mathrm{J}$ band has to be weighed by the limited associated SR. It appears that the $\mathrm{J}$ band photons spread more the focal spot than they increase the total flux. As a result, selecting $\mathrm{H}$ band alone is better than $\mathrm{J}+\mathrm{H}$. The selected spectral band is therefore $\mathrm{H}$ band.

To further concentrate the focal spot, two additional devices can be used:

- An ADC, which will compensate for the atmospheric dispersion, at the cost of a more complex setup and a loss in overall transmission. An ADC is made of two rotating prisms. We assume a total throughput loss due to reflection on the four diopters and to the absorption of the prisms $80 \%$. Moreover, the imperfect optical quality of these components originates a systematic residual dispersion of the spot. We considered a 5 mas residual dispersion. Last, the prisms must rotate with the pointing angle, which complexifies the system. Additional transport optics are probably mandatory to position the ADC in the appropriate plane. Phase 2 study shows that, with the mentioned assumptions, an ADC yields a limited gain, at least in photon noise regime ( 0.5 magnitude). We will not consider an ADC in the current baseline. 
- A dedicated DM, which will optimize the NGS spot quality (using tomographic high order information provided by the LGS WFSensors) at the cost of a more complex system and a minor loss in overall transmission. The opto-mechanical study showed that we can consider a micro DM mounted onto the NGS arm Tip Tilt platform. With this setup, the DM does not require a supplementary pupil plane, and the transmission loss, due to the transmission through the DM window, is estimated to $95 \%$. A dedicated DM will compensate for the residual anisoplanatism, and allow considering NGS with separation up to 1': without a dedicated DM, the SR for a NGS at 1' w.r.t. the optical axis is below $10 \%$. In the current baseline, we consider that a dedicated DM is mandatory.

- Finally, a new WFS concept is under study. Indeed, the best sensitivity in Tip Tilt is obtained with full-pupil WFS: it corresponds to a focalization of all the incoming photons on the smallest possible portion of a detector. In a focal plane sensor scheme, the four central pixels are used to compute the Tip Tilt, but the peripheral pixels can also be used to compute the other orders "for free", i.e. without degrading the noise propagation on the Tip-Tilt. First simulations show that this concept allows to sense low order modes with an error lower to the $125 \mathrm{~nm} \mathrm{rms}$ specified for magnitudes up to 17.5 (at the centre of the field), considering a 5e- read-out-noise detector. The associated sky coverage is larger than $60 \%$ at Galactic latitude of $60^{\circ}$.

The baseline NGS module contains two identical NGS arms. Each one features a dedicated DM and a focal plane wave front sensor working in $\mathrm{H}$ band at $500 \mathrm{~Hz}$. The WFS data are processed through a Kalman Filter. These specificities allow us to reach $60 \%$ sky coverage at Galactic pole. End-to-end simulation of the WFS on realistic phase screens is on-going.

\subsection{ATLAS control scheme}

Various control schemes are currently under investigation for ATLAS. Two major issues must be indeed addressed, first the wavefront reconstruction and correction by the DMs, in closed-loop, including a specific correction on the NGS WFS arms, then the mitigation of both LGS and NGS WFS measurements. The control scheme design must in addition balance performance with complexity.

So far, the main LTAO control law baseline is to consider Pseudo-Open Loop Control (POLC, [5]). Indeed, Fourier based tests have shown considerable performance improvement with MMSE tomographic reconstruction. Of course, in a dynamic system, LQG [6] would provide the optimal control solution. Still LQG presents a significantly increased complexity. POLC thus represents a satisfactory compromise between performance and complexity, associating a MMSE reconstruction to a 1st or 2nd order temporal control in control voltages space. Indeed, in the ATLAS context, the complexity of LQG would reach at least 4292 millions of additions/multiplications per iteration only for the M4/M5 control, while a POLC solution would represent only 416 millions of additions/multiplications per iteration (for comparison a standard least-square plus integrator control scheme would lead to 64 millions of additions/multiplications per iteration). However, various optimization of LQG control law are possible and will be considered in the future.

Another major issue is the LGS and NGS control mitigation. This covers both the issue of WFS measurements mitigation and control of $\mu$ DMs on the NGS WFS arms. From a pure reconstruction point of view, coupled tomography, in other words taking into account simultaneously the LGS and NGS WFS measurements to perform a global reconstruction, would represent the optimal solution. However this solution becomes tricky as soon as a particular handling of tip-tilt modes for windshake correction was considered, and that matrices updates were necessary. As a consequence, split tomography [7], in which both signal are separated and treated in two different tomographic processes, is considered as an alternative control scheme. This solution however may lead to poorer performance and the trade-off must be investigated further.

Finally, the original architecture of ATLAS leads to providing the NGS WFS arms with $\mu$ DMs, to ensure the correction of high-order modes in the NGS direction. In a somewhat similar fashion as MOAO, open-loop correction is thus foreseen, behind M4, in these directions, thanks to the LGS WFS measurements. The POLC control solution with split tomography is easily adapted to account for this prior correction, though an increase in complexity can't be avoided. 


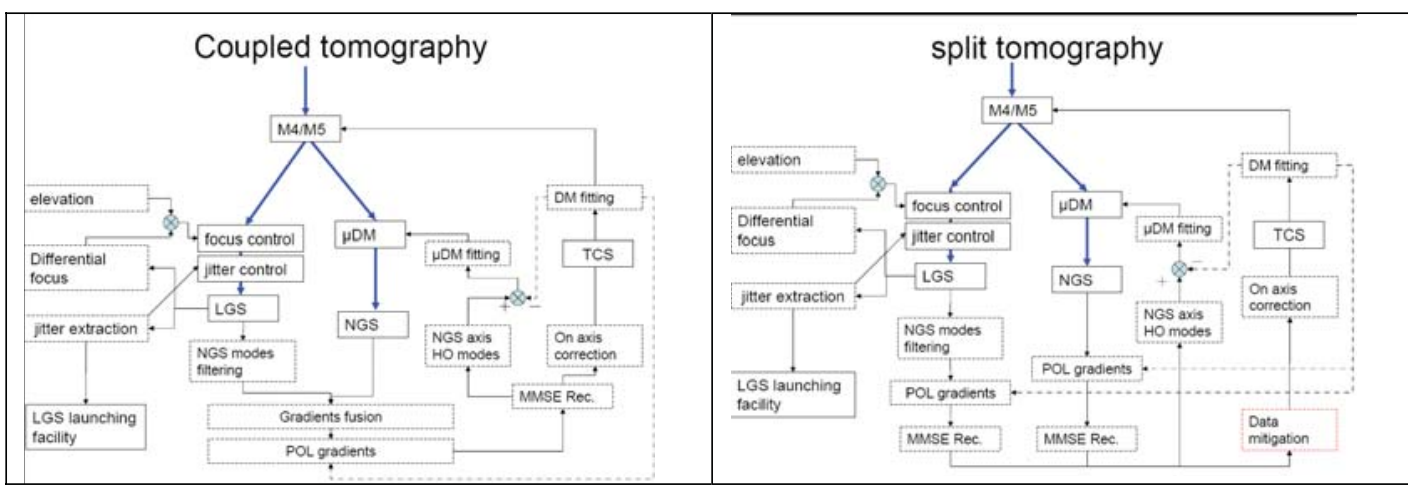

Figure 2 Concepts of ATLAS control. Left : Coupled tomography, Right split tomography

\section{Opto-mechanical design}

The main difficulty is to propose a design that fulfils the functional requirements without trespassing the volume and mass budgets allocated to the project. Six laser guide stars (LGS) and two natural guide stars (NGS) wavefront sensors are being considered.

An LGS is generated by focusing a high power laser in the Sodium layer of the atmosphere. Depending on the zenith angle of the telescope, the distance between the artificial source and the telescope is varying between 80 and $180 \mathrm{~km}$. This variation has an impact on two optical parameters: the LGS focusing $\left(3 \mathrm{~m}<\mathrm{F}_{\mathrm{LGS}}\right.$ $<8 \mathrm{~m})$ and the LGS footprint diameter at the entrance of the ATLAS envelope $\left(150 \mathrm{~mm}<\emptyset_{\mathrm{LGS}}<400 \mathrm{~mm}\right)$. To compensate for these effects, the opto-mechanical design of the six LGS sub-systems includes a compensation system that consists in a big-size optical zoom.

The two NGS wavefront sensors consist in two mechanical sub-systems able to aim for two distinctive targets in the 2 arcmin field of view at the focal plane of the telescope. In this setup, we want to collect as many photons as possible in the $\mathrm{H}$ band $(1.45 \mu \mathrm{m}<\lambda<1.85 \mu \mathrm{m}$ ), considering that NGS can be very faint sources (magnitude up to 20). In this purpose, the NGS opto-mechanical design integrates a high resolution deformable mirror with an ADC to best compensate for chromatism effects and $3^{\text {rd }}$ order optical aberrations such as spherical, coma and astigmatism.
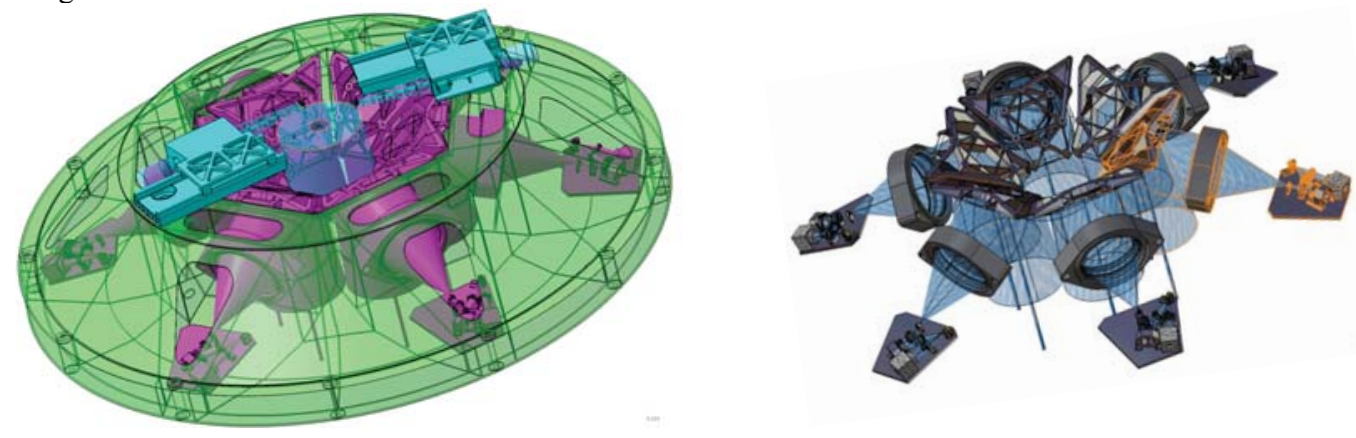

Figure 3 [left] ATLAS overall mechanical design (preliminary), in green the mechanical structure, in pink the LGS modules and in blue the NGS pick offs. The blue cylinder represent the 2 arcmin natural FoV. [Right] Optomechanicla design of the 6 LGS modules

This envelope aims at linking the ATLAS module to the telescope and the instrument to the ATLAS module. It has to be sufficiently stiff to satisfy this requirement. The dark blue part in the Figure 3 [left] is to be used to get back the optical field of any scientific instrument in order to measure the stability of the instrument in a referential linked to ATLAS. 


\section{ATLAS performance}

\subsection{Atmospheric conditions}

The main atmospheric parameters considered for the AO study are the following (with their ranges): a seeing of 0.8 "[0.6- 1.0'], an outer scale of $25 \mathrm{~m}$ [15-100m], a average wind speed of $16.3 \mathrm{~m} / \mathrm{s}[10-20 \mathrm{~m} / \mathrm{s}]$ and an isoplanatic angle (derived form a 9 layers $\mathrm{Cn}^{2}$ profile) of 2.08 "[ $[1.5-3$ "]. Note that we also considered an input signal due to windshake on one single tilt component of 300 mas.

\subsection{ATLAS nominal performance}

The ATLAS final error budget can be split in two main categories:

- the LGS high order budget gathering all the terms related to tomographic reconstruction

$$
\begin{aligned}
\sigma_{\text {res,high order }}^{2}= & \sigma_{\text {fit }}^{2}+\sigma_{\text {aliasing }}^{2}+\sigma_{\text {temp }}^{2}+\sigma_{\text {diff ref }}^{2}+\sigma_{\text {chrom }}^{2}+ \\
& \sigma_{\text {noise, LGS }}^{2}+\sigma_{\text {sodium structure var }}^{2}+ \\
& \sigma_{\text {tomo }}^{2}+\sigma_{C_{n}^{3} \text { model error }}^{2}+\sigma_{\text {noise model error }}^{2}+\sigma_{\text {DM saturation }}^{2}+\sigma_{\text {calibration error }}^{2}+\sigma_{\mathrm{NCPA}}^{2}
\end{aligned}
$$

with a global HO residual error of $260 \mathrm{~nm}$ rms

- the NGS budget gathering the tip-tilt and defoc residual error after their measurement and correction by the NGS $\sigma_{\mathrm{NGS}}^{2}=\sigma_{\text {temp/noise, } \mathrm{Z} 234}^{2}+\sigma_{\text {aniso }, \mathrm{Z} 234}^{2}+\sigma_{\text {other }}^{2}=125 \mathrm{~nm} \mathrm{rms}, \sigma_{\text {other }}^{2}$ gathers all the calibrations and model errors associated to the NGS.

A complete ATLAS simulation has been performed following the system design and the atmospheric parameters. The simulation tool is a Fourier-based model (see [8]) in which several modifications have been implemented to account for specific ATLAS issues (cone effects, tilt and focus indeterminations etc.). A final performance of $52 \%$ of SR in $\mathrm{K}$ on axis has been estimated with associated sky coverage larger than $60 \%$ (close to $80 \%$ ) at Galactic latitude of $60^{\circ}$. This leads to the following performance for various imaging wavelengths. Note that $100 \%$ sky coverage should be achieved at the price of a performance reduction. A PSF FWHM of 10 to 16 mas in the worst possible case is expected.

\section{Conclusion}

ATLAS is the LTAO module of the E-ELT. It aims at providing a diffraction limited PSF (SR around $50 \%$ in $\mathrm{K}$ band) in a small scientific FoV for a very significant part of the sky (more than $60 \%$ of the whole sky). 6 Laser Guide Stars (located on a 4.3 arcmin ring) will be used together with 2 Natural Guide Stars to be picked off in a 2 arcmin FoV. A MMSE-based RTC algorithm will be considered to obtain an optimal tomographic reconstruction of the turbulent volume and correct for Laser defects (cone effects). In parallel to the phase A design, some experimental studies are on-going at ONERA on the Wide Field AO bench (HOMER) in order to validate the LTAO concept and all its associated issues (WFS, tomographic reconstruction using optimal control law, calibrations) [9]. Finally, it is important to note that versatility and simplicity are two of the main drivers of the system design. It should be compatible with various scientific instruments and should remain as simple as possible (from an opto-mechanical point of view) in order to be implemented in the first generation of the E-ELT instrumentation.

\section{References}

1. B. Crépy et al., (EDP Sciences, this conference, 2009)

2. D. Gallieni et al., (EDP Sciences, this conference, 2009)

3. J. Barriga et al., (EDP Sciences, this conference, 2009)

4. E. Diolaiti at al., (EDP Sciences, this conference, 2009)

5. L. Gilles, Applied Optics, 44, 6, 2005.

6. C. Petit et al., J. Opt. Soc. Am. A, 26, 1307-1325, 2009

7. L. Gilles et al., (EDP Sciences, this conference, 2009)

8. B. Neichel et al., Proc. SPIE, vol. 7015 (Marseille, 2008)

9. A. Costille et al., Opt. Soc. Am. A, to be published 